\title{
La creatividad y el capital intelectual
}

Creativity and intellectual capital

Criatividade e capital intelectual

Laura Elizabeth Cervantes Benavides

Universidad Autónoma de Ciudad Juárez, México

lauraeliz55@hotmail.com

\section{Resumen}

La creatividad tiene una relación directa con el capital intelectual, todas las personas lo poseen, pero se piensa que sólo los que han adquirido una educación formal y han alcanzado una especialización, son capaces de aplicarlo para desarrollar productos teóricos: conceptos, nuevas teorías, nuevo conocimiento, procedimientos, etc., productos tecnológicos: máquinas, aparatos, nuevos materiales, innovaciones, etc., la creatividad es un componente cognoscitivo esencial del pensamiento, se puede enseñar con técnicas, y el resultado se puede utilizar en la vida cotidiana. Palabras clave: creatividad, capital intelectual, estrategias creativas, aprendizaje creativo.

\section{Abstract}

Creativity has a direct relationship with the brainpower, all people possess, but it is thought that only those who have acquired a formal education and have achieved specialization, are able to apply it to develop theoretical products: concepts, new theories, new knowledge, procedures, etc., technological products: machines, equipment, new materials, innovations, etc., creativity is an essential component of cognitive thinking can be taught techniques and the result can be used in everyday life.

Key words: creativity, intellectual capital, creative strategies, creative learning. 


\section{Resumo}

Criatividade tem uma relação direta com os recursos intelectuais, todas as pessoas possuem, mas acredita-se que apenas aqueles que adquiriram uma educação formal e ter alcançado a especialização, são capazes de aplicá-la a desenvolver produtos teóricos: conceitos, novas teorias, novo conhecimentos, procedimentos, etc., produtos tecnológicos: máquinas, equipamentos, novos materiais, inovações, etc., a criatividade é um componente essencial do pensamento cognitivo podem ser ensinadas técnicas eo resultado pode ser usado na vida cotidiana.

Palavras-chave: criatividade, capital intelectual, estratégias criativas, de aprendizagem criativa.

$\begin{array}{llll}\text { Fecha recepción: Enero } 2016 & \text { Fecha aceptación: Junio } 2016\end{array}$

\section{INTRODUCCIÓN}

Todos los días nos enfrentamos a situaciones problemáticas, que nos demandan alternativas para solucionar el o los problemas. Con la misma frecuencia nos damos cuenta que la manera en que concebíamos una solución de un problema, en un momento determinado, ya no era adecuada para solucionarlo, en otro momento, debido a la complejidad de la dinámica económica y social. Frente a esta realidad, las organizaciones educativas se encuentran en la búsqueda de nuevos métodos para llevar a cabo el proceso de enseñanza-aprendizaje y, dentro de estos métodos, enseñar habilidades creativas, para que los estudiantes sean capaces de aprender (método de casos, educación basada en problemas, educación constructivista, educación por competencia, etc.). En la actualidad, son pocas las propuestas para la enseñanza de las habilidades creativas, dentro del mismo contenido curricular que se les enseña a los estudiantes.

El gran problema que enfrenta la educación es que no se ha logrado alcanzar el objetivo primordial: "La formación de individuos críticos, que utilicen su creatividad y sean conscientes que son poseedores de un capital intelectual, para proponer soluciones adecuadas a los problemas que enfrenta el entorno". Al analizar esta situación que enfrenta el sistema educativo, se puede identificar que, en la mayoría de los sistemas de educación tradicionales, se siguen desarrollando programas, cartas descriptivas, desarrollando habilidades o competencias y moldeando actitudes, 
integrados fundamentalmente con contenidos que no permiten alcanzar el objetivo antes mencionado.

Los contenidos descritos en los documentos, no incluyen elementos instruccionales que indiquen una práctica deliberada, que permita estimular las formas de pensamiento lateral. En cambio, los contenidos que se han identificado, derivan en complementar los esquemas de comportamiento lineal que tradicionalmente se aprenden para solucionar los problemas.

Con la descripción de esta situación problemática, que enfrenta actualmente la educación, nos obliga a todos los docentes que estamos frente a grupo, a redefinir nuestro objetivo programático en la enseñanza cotidiana. ¿Qué tipo educación debe darse al estudiante, para enseñarle a relacionar el proceso de creatividad con el capital intelectual? El objetivo es describir la relación procedimental que existe entre la creatividad y el capital intelectual. Para darle respuesta a esta interrogante, se realizó una descripción analítica, para proponer que: un aprendizaje procedimental, facilita la enseñanza de la creatividad al estudiante, y deriva en relacionar el producto creativo como una posesión de su capital intelectual.

El trabajo que se presenta, es el resultado de las observaciones que se hicieron a estudiantes de los niveles de preparatoria, universidad, y grupos de empresarios, con el método procedimental, de educación Basada en Procesos Cognoscitivo, como una propuesta de los estudios sobre los procesos de pensamiento. Representa una síntesis de los Programas de Aprender a Pensar y de Desarrollo de Habilidades Creativas.

Para facilitar la explicación de la manera de desarrollar la creatividad para relacionarla con el capital intelectual, se presenta en los siguientes apartados: el apartado uno, "la creatividad y su relación con los productos mentales", en este apartado se describe la forma en que de manera procedimental se deriva en los productos mentales; el apartado dos, "el proceso enseñanza aprendizaje para la creatividad", en este apartado se explica la operacionalización de la enseñanza, para facilitar el aprendizaje, destacando la importancia para estimular el acto creativo; el tercer apartado, "el capital intelectual y su relación con la empresa", se aborda las forma en la cual se lleva a cabo la relación entre la capacidad de creatividad y su aplicación para la solución de problemas en la empresa. Por último, incluimos una conclusión, en la cual intentamos integrar lo desarrollado en los apartados previos.

\section{MÉTODO}




\section{La creatividad y su relación con los productos mentales}

La creatividad es la habilidad metacognoscitiva que permite al ser humano generar alternativas novedosas y originales para solucionar todo tipo de problemas. Otra definición sencilla, explica que la creatividad es la capacidad de realizar una producción a la vez novedosa o adaptada (Olivier, Houde, 2003). La creatividad explicada como esencia humana, sigue siendo estudiada, y conocer la forma para develarla y enseñarla, aunque no todos los resultados han sido concluyentes, han permitido identificar el comportamiento de las personas creativas, sobre todo en el momento cuando activa el operador mental, que activa el acto creativo. Bajo este supuesto, mostraremos una manera que la creatividad, se puede enseñar, se debe practicar, se puede internalizar, se puede mejorar, y se puede convertir a un estudiante en un ser creativo.

En los primeros estudios se han integrado definiciones y elementos conceptuales sobre los rasgos relevantes de la creatividad, o de la persona creativa, también se han identificado discrepancias y diferencias de los mismos, (Osborn 1955, Gordon 1961, Helsein 1969, Rouquette 1977, Crawford 1977, De Bono 1983, Perkins 1981).

Todos estos trabajos han permitido elaborar la propuesta cognoscitiva de la creatividad, en la parte procedimental y actitudinal, como un intento para integrar el pensamiento inventivo, con las funciones específicas del pensamiento lineal y lateral, para identificar el tipo de las habilidades que se pueden desarrollar. En la propuesta de Sánchez Amestoy, se sintetizan todos los trabajos arriba mencionados sobre los rasgos relevantes de la creatividad. Tabla I

El trabajo que se presenta es una continuación del trabajo de campo, sobre la enseñanza procedimental de los procesos mentales, realizado por Margarita Amestoy de Sánchez durante la década de los ochenta y noventa. Los resultados concluyen que la creatividad tiene un componente, que es el resultado de las experiencias acumuladas en el individuo, que se manifiestan de manera inconsciente, y que, en un momento dado, afloran y se manifiestan como productos creativos. 
Tabla I. Características esenciales en creatividad En la persona y habilidad

\begin{tabular}{|c|c|}
\hline $\begin{array}{c}\text { Características } \\
\text { en la persona }\end{array}$ & $\begin{array}{c}\text { Tipo de habilidad para } \\
\text { desarrollar para: } \\
\text { percibir un problema }\end{array}$ \\
\hline flexibilidad & plantear un problema \\
\hline sentido del humor & $\begin{array}{c}\text { plantear y verificar } \\
\text { hipótesis }\end{array}$ \\
\hline fluidez & $\begin{array}{c}\text { definir y redefinir } \\
\text { problemas }\end{array}$ \\
\hline movilidad & $\begin{array}{c}\text { generar productor } \\
\text { originales }\end{array}$ \\
\hline $\begin{array}{c}\text { es objetiva y } \\
\text { subjetiva }\end{array}$ & generar innovaciones \\
\hline $\begin{array}{c}\text { piensa en } \\
\text { resultados }\end{array}$ & \\
\hline
\end{tabular}

Elaboración propia

Por lo tanto, la creatividad como proceso mental que, se puede enseñar, se puede desarrollar, si se desarrollan habilidades metacognoscitivas en los estudiantes (preguntas internas que nos hacemos: ¿cómo aprendí lo que me enseñaron?,¿esto que aprendí, dónde lo puedo aplicar?, ¿cómo transformo el entorno con este conocimiento?) la posesión de estas habilidades en los estudiantes, permiten generar un "producto mental" nuevo, diferente, único y original, como resultado de una forma de actuar y pensar que tiene nuestro cerebro, porque le facilita procesar la información de manera diferente, a la forma lógica que se le ha enseñado a procesarla, de tal manera, que el resultado se manifieste como una producción intelectual, que transforma las situaciones, con los productos mentales, generados con ideas novedosas, objetos únicos, objetos innovadores, o inventos que puedan transcender o transformar la realidad del individuo, cuando se le enseña: un activador mental, estimular el acto creativo, el control y la retroalimentación de su producción intelectual.

\section{RESULTADOS}

\section{El proceso enseñanza aprendizaje para la creatividad}

La creatividad tiene una relación directa con la generación de productos mentales. El proceso para enseñar y aprender creatividad, con el enfoque procedimental y sistémico ${ }^{1}$ desencadenando

\footnotetext{
${ }^{1}$ Este enfoque es el resultado del trabajo de estudio de campo, sobre el estudio de los procesos de pensamiento desarrollado por Margarita A. de Sánchez, 1983-1984 y junto con los trabajos realizados con Eduard de Bono, tiene su base en las tres propuestas que se presentaron al Ministerio de Inteligencia de Venezuela: sacar al individuo de
} 
los activadores de la creatividad, para estimular el acto creativo, cuidando los ambientes en el que se genera la creatividad, controlando y retroalimentando el proceso.

\section{Activadores de la creatividad}

El activador mental ${ }^{2}$ (Margarita A. de Sánchez, 1990) es un mecanismo que enciende el funcionamiento el acto mental, en el programa Aprende a Aprender, se le conoce como el operador mental, Considere Objetivos o Posibilidades, (COP). Algunas personas nacen con esa habilidad que les permite activar su creatividad, otras personas pueden aprender a desencadenar el mecanismo. Una vez que aprenden a desencadenarlo, puede dar origen a una producción creativa.

Si esto ocurre en las personas creativas, se puede enseñar y estimular la mente de los estudiantes, para que aprendan de manera consciente el proceso del acto creativo, primero para practicar el proceso y después para que lo internalice a través de patrones especiales de pensamiento, para que actúen como activadores de creatividad, creando un nuevo ambiente que permita la generación de nuevas ideas, reorganizando y cambiando los patrones tradicionales.

Los activadores de creatividad producen conexiones de patrones de pensamiento entre los existentes, con información nueva y contribuyen a formar nuevos patrones de pensamiento, estas conexiones permiten aumentar la posibilidad de integración para la generación de ideas, unas originales y otras innovadoras, cuando estas ideas se relacionan, integran un nuevo patrón de pensamiento, integrado por nuevas ideas, ideas no convencionales, ideas con otros enfoques, derivados de la inclusión de toda la información. Para enseñar la habilidad creativa en los estudiantes, el modelo sistémico propone separar el acto creativo en tres momentos: entrada, proceso y producto.

\section{Estimular el acto creativo}

El acto creativo es una habilidad que todos los seres humanos lo poseemos, de esta forma, generamos todo tipo de productos mentales, unos sencillos, otros complejos, otros destacan por diferentes a los convencionales, por ejemplo: de arte, música, pintura. Todos los productos creativos, son generados a través del acto creativo, para luego ser elaborados, concretarlos o

los extremos polarizadas para explorar nuevos caminos, utilizar la información de manera no convencional y reestructurar sus patrones de pensamiento.

2 El activador mental es un operador mental. Véase Considere Objetivo o Posibilidades (COP). 
generalizándolos; pueden ser los productos para uso diario, productos para la salud, productos teóricos (conceptos, nuevas teorías, nuevo conocimiento, procedimientos, etc.) productos tecnológicos (máquinas, aparatos, nuevos materiales, innovaciones, etc.).

En algunos casos el acto creativo es espontáneo, o planificado, existen personas que lo generan, pero no lo ordenan (imaginación), otras que lo hacen y tienen una organización mental propia que les permite aprovechar su potencial y lo concretan. Todas las personas tienen la capacidad de realizar acciones a través de un acto creativo, pero, cuando se piensa en ¿cómo se genera ese acto creativo? se llega a una encrucijada, porque se tiene la certeza, que sólo lo hacen los genios, los inventores con ideas fuera de lo común, o los seres dotados de una súper memoria.

Lo que observó en los estudios de campo, permitió operacionalizar este proceso de pensamiento, para estandarizarlo y enseñarlo de manera consciente, así se empezó a enseñar a los estudiantes, con una técnica que permite practicarla, formar patrones mentales nuevos, hasta poseer el hábito del pensamiento creativo, y pasó de ser la creatividad "espontánea" para convertirse en la creatividad aprendida, con una enseñanza deliberada, para convertirse en la creatividad. Para estimular el acto creativo se requieren tres etapas: la entrada, el proceso y el producto.

\section{El acto creativo: la entrada}

En esta etapa es necesario 1) identificar el problema (proceso mental identificación de problema, véase proceso, en Margarita, A. de Sánchez) como resultado de la descripción de la realidad, en ésta, es necesario elaborarla a partir de los eventos de la observación directa y la observación indirecta, eliminando toda inferencia y experiencia previa, de tal manera que la identificación del problema derive en la pregunta de investigación. 2) identificar la cantidad de información disponible, para darle respuesta a la pregunta que se plantea., 3) operacionalizar las variables que lo afectan.

\section{El acto creativo: el proceso}

Durante el proceso de creatividad se utilizan diferentes acciones, se empieza por: 1) las estrategias y técnicas utilizadas, como generadoras o de "técnicas detonantes" (técnica del trampolín, técnicas de la cadena, técnicas de la pregunta) para generar alternativas novedosas., 2) el número de alternativas novedosas de solución, 3) el resultado esperado, 4) la secuencia del proceso y 5) la representación del resultado. 


\section{El acto creativo: el producto}

En la etapa del producto del acto creativo, es importante 1) comunicar el resultado (oral y escrita) para que sea elaborado y, por último, 2) se presenta el producto final, para darle solución a la pregunta planteada y la que desencadenó el activador creativo. Tabla II.

Tabla II. Estimular el acto creativo

\begin{tabular}{|l|}
\hline La entrada: \\
Identificar el problema. \\
Identificar la cantidad de información \\
disponible \\
La operacionalización de las variables que \\
lo afectan \\
\hline El proceso \\
Las estrategias y técnicas utilizadas \\
Número de alternativas de solución \\
El resultado esperado \\
La secuencia del proceso \\
El tipo de representación \\
\hline El producto \\
Comunicar el resultado \\
Presentar el producto \\
\hline
\end{tabular}

Elaboración propia

\section{Identificar el ambiente psicológico y físico}

El ambiente es un factor que constituye un factor de desempeño, influye y modifica de manera decisiva la producción creativa del estudiante, por esta razón el maestro debe prepararse para enseñarle a "parir la idea", porque esa solución es del estudiante. (una explicación metafórica sería que el maestro sólo ayuda a recibir e integrar la idea, del mismo modo que una partera, ayuda a recibir el bebé de la madre, pero el bebé jamás será de la partera).

Los dos ambientes son elementos que van a influir en los efectos, modificaciones, o cambios en el producto, con ayuda del maestro, el estudiante también puede generar nuevas entradas, cerrar, activar o desactivar el acto creativo, en este proceso de retroalimentación y monitoreo, la responsabilidad que tiene el maestro en el ambiente es determinante., sintetizando lo que sugiere Raymon N. Hatch y James W. Costar (1990,) la intervención del maestro, "permite modificar el ambiente, proporciona la información, adapta las técnicas en función de las necesidades, es un 
autoridad, trabaja con los resultados (...), evalúa y contribuye a la organización” de tal forma que el ambiente no afecte la concreción del acto creativo del estudiante. De tal forma que el maestro pueda guiar al estudiante para la generación de nuevo conocimiento y la posesión de su capital intelectual. Por esta razón el maestro jamás podrá ser sustituido por una "clase virtual" cuando se pretende sustituir este proceso cognoscitivo.

\section{Controlar el proceso}

Una vez que se ha gestado el producto como resultado del acto creativo. El control del proceso creativo, permite integrar el metaproceso, para operacionalizarlo, hacer uso de trasmisión de la información y propicia la efectividad de los resultados, para su aplicación y transferencia.

\section{Retroalimentrar los mecanismos creativos}

La retroalimentación en el proceso de creatividad, es un mecanismo que se enseña al estudiante para que aprenda a mejorar el resultado de su propio producto mental, de tal forma que empieza a adquirir y practicar su propio aprendizaje deliberado, para que éste procedimiento de creatividad, pase a convertirse en un proceso cognoscitivo interno, le permita al estudiante cambiar sus propios patrones lógicos, por los creativos a través de la práctica deliberada.

La retroalimentación del proceso de creatividad, se vuelve automática, y le permite al estudiante alcanzar un efecto regenerativo y dinámico para que, de manera consciente, influya en el pensamiento del estudiante y este a su vez en la transformación del ambiente.

El objetivo final de la retroalimentación en el proceso creativo es para que el estudiante adquiera un mecanismo que propicie el enfrentamiento del estudiante consigo mismo y con su actuación pensante; esta actuación le permite la validación de estándares internos de cada tarea ejecutada, le permite aplicar los correctivos o ajustes que generan autoregulación y flexibilidad, de las acciones realizadas de manera consciente, y contribuyen a mejorar su proceso de creatividad y a perfeccionar los resultados. Así el estudiante puede confrontar los resultados obtenidos en los niveles prefijados, de aspiraciones y de exigencias planteadas, sin necesidad de que se le evalué de manera externa. 


\section{Proceso de creatividad}

1. Activador mental

2. El acto creativo

3. El control del proceso

4. La retroalimentación

\section{DISCUSIÓN}

\section{El capital intelectual y su relación con la empresa}

El capital intelectual es un factor económico, que le genera valor a los productos y actividades de la empresa, como capital intelectual, las personas que utilizan la creatividad aprendida de manera procedimental, son conscientes que puede ser estimulada, para desarrollarla como una habilidad cognoscitiva, para convertirla en un capital intelectual, que es un elemento fundamental del factor económico humano, que permite darle solución a los problemas, generar novedosos productos y hacer más cómoda la vida del ser humano.

Pero, si ese producto mental que generó el estudiante a partir del proceso de la creatividad, no se le enseña la forma en que se debe realizar su aplicación y transferencia (Procesos de Aplicación y Transferencia), no se puede convertir en capital intelectual de su creador (el estudiante) y no se logra concretar el objetivo final que es vincular la Escuela con la Empresa.

La importancia de enseñar al estudiante la aplicación y la transferencia de su propio producto mental, es que se sienta poseedor de un capital intelectual, que le va permitir poseer un factor económico, generador de valor, que le va a permitir obtener una utilidad económica, dentro de una empresa, o de manera independiente.

La persona emprendedora sabe transformar su creatividad en capital intelectual, porque es una persona que sabe que el producto mental que generó, es una idea de negocio que a su vez creará una nueva actividad económica, cuando esta idea se transfiere y se aplica en la forma de inventos, que facilitan la vida a toda la humanidad, y no dejar a la suerte, como muchos de ellos han ocurrido y que "hoy día resulta difícil imaginar la vida sin estas grandes ideas.

Tales como armas, herramientas, fuego, rueda, teléfono, juegos juguetes, ajedrez, ropa, escritura, etc." (Marcelo, Duarte, Grijalbo, 1995) y que han generado un valor monetario muy importante 
para quienes fueron capaces de venderlos, creadores de ese producto o simplemente emprendedores de una actividad económica, sin haber gestado el producto mental.

La creatividad puede explicase de manera sencilla con la inventiva de Leonardo Da Vinci, que inventó casas portátiles desmontables, máquinas laminadoras, un aparato para cortar tornillos, un mecanismo para hilar y una draga para puertos. Da Vinci, fue el primer hombre que montó la aguja magnética, en un eje vertical, dándonos la brújula tal y como hoy la conocemos e inventó lo que llamamos en la actualidad piñón de diferencial y un anemómetro o medidor de viento. (Donald, Culross, Peattie, 1986)

Un ejemplo cotidiano y que nos explica la forma en que se utiliza la creatividad, es cuando se resuelven conflictos en el extranjero, este caso implica una gran cantidad de reglas y alternativas para resolver el conflicto. Los hechos reales, indican información que indican una regla directa: integrada con una relación jurídica y un derecho aplicable (consecuencia jurídica). Pero al mismo tiempo, se requiere buscar una regla indirecta, a través de un punto de conexión. (Ricardo, R. Balestra, 1995) en este caso, la mejor solución, tiene su base en la creatividad.

La Creatividad y su relación con El Capital Intelectual

Situación

problemática

\section{CREATIVIDAD}

Productos mentales

Solución de problemas

\section{CAPITAL INTELECTUAL}

Aplicación y Transferencia

Empresa 


\section{CONCLUSIÓN}

El reto que enfrentan las organizaciones educativas en la actualidad, es formar profesionistas capaces de resolver problemas, enseñar el proceso de creatividad y desarrollar estas habilidades a través de la práctica deliberada. La creatividad tiene una relación directa con los productos mentales, y los productos mentales es una esencia humana.

Los productos mentales pueden ser concebidos, pero no siempre son materializados, para esto, se requiere un proceso enseñanza aprendizaje, para enseñar la creatividad, un maestro que dirija, retroalimente, verifique y ayude a activar las ideas a los estudiantes, través de las diferentes estrategias de creatividad.

La posesión consciente del proceso de creatividad, se traducirá en la posesión consciente de un capital intelectual, un capital intelectual que se convierte en factor económico, que es el origen del nuevo valor que requieren las empresas, para conservar su competitividad en esta dinámica globalizadora. "Se debe iniciar el magnífico florecimiento intelectual que (...) llamamos ciencia", imitando a Sócrates que encaminó el método científico al estudio del arte de vivir" (Max, Easman, 1986) Con este legado, debemos enseñar a los estudiantes que son poseedores de un capital intelectual, que les permitirá vivir, con comodidad, una vez que conozcan la importancia de este factor económico dentro de las empresas. Los productos derivados de la creatividad y derivados como capital intelectual, no se puede automatizar fácilmente, aun cuando se pueda enseñar la operacionalización, por ello es necesaria la intervención del maestro. 


\section{Bibliografía}

Balestra, Ricardo, R (1995) Empresas trasnacionales, inversiones extranjeras y arbritaje, Buenos Aires.Ed. Abeledo-Perrot.

Culross, Peattie, Donald, (1986) "El hombre más justo, más bueno y más sabio", en, Genios y Figuras, Tomo II, cuarta reimpresión. México. Ed. Reader’s Digest

Duarte, Marcelo, (1995) Guía de curiosos, México. Ed. Grijalbo.

Easman, Max, (1986) "El hombre más justo, más bueno y más sabio”, en, Genios y Figuras, Tomo I, cuarta reimpresión, México. Ed. Reader's Digest.

Gregory Richard, (1995) Diccionario Oxford de la mente, Madrid. Alianza editorial,

Grupo Balpe (1993) Intelector 3 El poder de la mente, desarrolle su creatividad, México. Ed. Grijalbo.

Houdé, Olivier, et al, (2003) Diccionario de Ciencias cognitivas, México, Amorrotu editores.

N. Hatch, Raymon y Costar W. (1990) Dirección de grupos, Tomo V, Ediciones ciencia y técnica, S.A. México. Editorial Limusa.

Sánchez, Margarita A., (1995a) Desarrollo de Habilidades del Pensamiento, Creatividad, México. Ed. Trillas

Sánchez, Margarita A., (1997b) Desarrollo de Habilidades del Pensamiento, Procesos Directivos, Ejecutivos y de Adquisición de Conocimiento, México. Ed. Trillas 